\title{
A Method for Scheduling Air Traffic with Uncertain En Route Capacity Constraints
}

\author{
Heather Arneson* \\ University of Illinois at Urbana-Champaign, Urbana, IL 61801, USA \\ Michael Bloem ${ }^{\dagger}$ \\ NASA Ames Research Center, Moffett Field, CA 94035-1000, USA
}

\begin{abstract}
A method for scheduling ground delay and airborne holding for flights scheduled to fly through airspace with uncertain capacity constraints is presented. The method iteratively solves linear programs for departure rates and airborne holding as new probabilistic information about future airspace constraints becomes available. The objective function is the expected value of the weighted sum of ground and airborne delay. In order to limit operationally costly changes to departure rates, they are updated only when such an update would lead to a significant cost reduction. Simulation results show a $13 \%$ cost reduction over a rough approximation of current practices. Comparison between the proposed asneeded replanning method and a similar method that uses fixed frequency replanning shows a typical cost reduction of $1 \%$ to $2 \%$, and even up to a $20 \%$ cost reduction in some cases.
\end{abstract}

\section{Nomenclature}

$\Delta t \quad$ Duration of time step.

$N \quad$ Number of sections in the route leading from the airport to the FCA.

$S \quad$ Number of weather scenarios.

$c^{s}(k) \quad$ Expected capacity of weather avoidance route (i.e. the number of aircraft that can enter the weather avoidance route during time step $k$ ) under weather scenario $s$.

$p^{s} \quad$ Probability that scenario $s$ will occur, $\sum_{s=1}^{S} p^{s}=1$.

$\tau_{i} \quad$ Number of time steps required for aircraft to traverse section $i$.

$x_{i}^{s}(k) \quad$ Total number of aircraft in section $i$ at the end of time step $k$ under weather scenario $s$.

$\bar{x}_{i} \quad$ Maximum capacity of section $i$.

$h_{i}^{s}(k) \quad$ Total number of aircraft holding at section $i$ at the end of time step $k$ under weather scenario $s$.

$\bar{h}_{i} \quad$ Maximum holding capacity of section $i$.

$u_{i}^{s}(k) \quad$ Net number of aircraft sent into or removed from holding at section $i$ during time step $k$ under weather scenario $s$.

$q_{i}^{s}(k) \quad$ Number of aircraft flowing out of section $i$ during time step $k$ under weather scenario $s$.

$\mathcal{D}(k) \quad$ Number of originally scheduled departures during time step $k$.

$d(k) \quad$ Number of controlled departures during time step $k$.

$K_{F} \quad$ Final time step of the full plan.

$K_{I} \quad$ Initial time step of full plan, $K_{I}<K_{F}$.

$K \quad$ Start time step of current plan, $K_{I} \leq K<K_{F}$.

$\hat{T} \quad$ Accurate forecast horizon, integer multiple of $\Delta t$.

$\hat{K} \quad$ Accurate forecast horizon, integer number of time steps, $\hat{K}=\frac{\hat{T}}{\Delta t}$.

$K_{R} \quad$ Replanning frequency, $K_{R} \leq \hat{K}$.

$w_{g} \quad$ Weight applied to ground holding in the calculation of total cost.

$w_{a} \quad$ Weight applied to airborne holding in the calculation of total cost.

\footnotetext{
*Graduate Student, Aerospace Engineering, 306 Talbot Lab/MC-236, 104 S Wright St., Urbana, IL 61801.

$\dagger$ Research Aerospace Engineer, Systems Modeling and Optimization Branch, Aviation Systems Division, NASA Ames Research Center, MS 210-10, Moffett Field, CA 94035-1000.
} 


\section{Introduction}

When the capacity of en route airspace is limited due to convective weather or other factors, the traffic through this area must often be delayed to prevent capacity from being exceeded. Weather conditions in recent years have caused approximately $65 \%$ of delays, ${ }^{1}$ so this is an important problem. The term used to refer to a region of airspace with reduced capacity is Flow Constrained Area (FCA). Flights scheduled to fly through the FCA are identified and given an option of routing around the FCA. Flights that choose to travel through the FCA must be controlled so that the FCA capacity is not exceeded. Recent research has focused on the development of algorithms for scheduling ground and airborne delay. ${ }^{2,3}$ With the inherent uncertainty in weather forecasts, and thus in FCA capacity predictions, an important aspect of any scheduling algorithm is the way in which this uncertainty is handled.

Scheduling algorithms have accounted for this uncertainty in a variety of ways. Bertsimas and Stock Patterson assume a deterministic capacity model in Ref. 4 and note that more work must be done to account for the uncertainty in en route capacities dependent on weather. Hoffman et al. ${ }^{2}$ present a method of scheduling flights for use in an AFP that is based on en route resource allocation. Here, as in Ref. 4, the authors mention considering for future work the stochastic effects of uncertainty in airspace capacity. In Ref. 3 , Krozel et al. assume that the weather forecast will be accurate for some amount of time, beyond which the forecast is uncertain. The authors propose rescheduling at a fixed frequency that ensures a reschedule occurs before the planning horizon extends into the uncertain time. In testing their algorithm, they use more or less severe weather predictions, as compared to the actual weather, beyond the accurate forecast horizon to obtain a rough measure of the robustness of their algorithm to uncertainty in weather predictions. However, their algorithm does not systematically consider various weather forecasts and their relative probabilities.

In this work, probabilistic forecasts of FCA capacity are incorporated into scheduling. Furthermore, a cost-benefit analysis is proposed to determine when departure rate replanning should occur. A discrete time aggregate flow model, similar to the cell transmission model, ${ }^{5}$ describes the flow of aircraft through a network of airspace. This solution method is iterative. It involves solving two linear programs (LPs). One solves for new departure rates and for airborne holding. The other fixes departure rates at their current values and solves for airborne holding. Updated departure rates from the first LP are implemented only when they lead to a significant cost reduction. Both LPs consider probabilistic airspace constraint forecasts. The cost function is the expected value of a combination of the ground holding cost and the cost of airborne holding. These LPs are solved repeatedly throughout the day with updated FCA capacity forecasts.

Background is provided in Section II. The problem is stated and the solution method is then described in Section III. Simulations using synthetic data compare this approach to simpler approaches that use only one capacity profile to represent predicted FCA capacity. Simulations designed to study the effects of changing the cost of replanning on the total cost as compared to replanning at a fixed frequency were performed as well. Simulation results are reported in Section IV. Conclusions and future work are discussed in Section V.

\section{Background}

Currently, the maximum flow through an FCA is set by air traffic controllers based on their experience controlling traffic in that region during similar weather events. A more systematic use of probabilistic forecasts of the capacity of an FCA may allow for better utilization of airspace and greater FCA throughput. This possibility is investigated in this research. Therefore, it is focused on the use of probabilistic airspace capacity forecasts as opposed to the generation of these forecasts.

Some probabilistic weather forecasts exist and are a current topic of research. For instance, the National Convective Weather Forecast (NCWF-2) provides a probabilistic forecast for the NAS with a 0 to 2 hour range. A version of this forecast, NCWF-6, extends the forecast to 6 hours. The Collaborative Convective Forecast Product (CCFP) is a 2, 4, and 6 hour forecast product that is probabilistic in the sense that each predicted weather polygon is assigned a low or high confidence. ${ }^{6}$ A more recent product known as the Localized Aviation MOS Program (LAMP) provides probabilities of convection in $20 \mathrm{~km}$ by $20 \mathrm{~km}$ grid cells up to 25 hours in the future. ${ }^{7}$

Weather forecasts must also be converted into predictions of flight deviations to be useful for traffic flow management (TFM) decision making. The Convective Weather Avoidance Model (CWAM) ${ }^{8}$ uses deterministic weather forecasts from the Corridor Integrated Weather System (CIWS) ${ }^{9}$ to predict pilot deviations around weather. More specifically, CWAM produces polygons containing weather and specifies the proba- 
bility that pilots will deviate around each polygon.

Another aspect of determining airspace capacity due to weather is finding not just the probability of flight deviations, but also a deterministic or probabilistic forecast of the resulting amount of traffic flow that can pass through a particular type of airspace. This sort of computation involves the structure of the airspace that is not passable due to weather. For example, the use of a scenario-based stochastic weather model to generate expected capacity and a probability distribution of capacity for an FCA is proposed in Ref. 10. For each weather scenario, the authors use a mincut algorithm, similar to the algorithm presented in Ref. 11, to generate a set of routes through the FCA which avoid convective weather. A capacity forecast and probability are associated with the weather avoidance routes generated for each weather scenario. Thus probabilistic capacity profiles for weather avoidance routes through the FCA are generated from probabilistic weather forecasts. As suggested by the authors of Ref. 10, other route generation algorithms, such as the Flow-Based Route Planner ${ }^{12}$ which generates routes through an FCA with deterministic constraints or the scanning method, ${ }^{13}$ could be extended to work with stochastic weather models.

The research in this paper is built on an approach similar to that presented in Ref. 10, in which uncertainty in weather predictions gets translated into several different possible capacity profiles for each weather avoidance route through an FCA, each with an associated probability of occurring. An example set of capacity profiles for a weather avoidance route through an FCA during a weather event is shown in figure 1. Associated with the capacity forecast is an accurate forecast horizon, $\hat{T}$. The accurate forecast horizon is the length of time over which the weather forecast is assumed accurate, and thus the predicted capacity of the weather avoidance route is assumed accurate. All capacity profiles are identical over the accurate forecast horizon. Beyond this horizon, the capacity profiles may differ. Each profile has a probability that corresponds to uncertainty in the weather forecast.

Finally, much research has been done on both static and dynamic and both deterministic and stochastic management

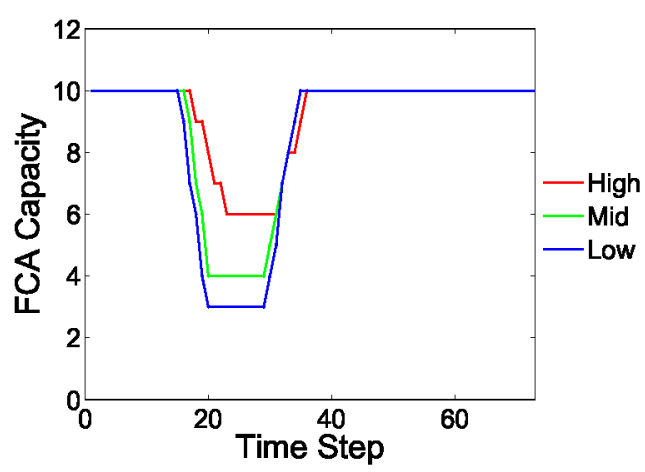

Figure 1. Example of capacity prediction for a single weather avoidance route through the FCA given as three possible capacity profles with associated probabilities. The FCA capacity has units of number of aircraft per time step. of Ground Delay Programs (GDPs), ${ }^{14,15}$ which constrain arrivals to weather-impacted airports while also considering equity. This line of research has not yet been extended to the management of weather-induced congestion in airspace, even though much of it may be directly applicable. Additionally, some work has been done to find scenarios for airport acceptance rates with corresponding probabilities from historical traffic data, ${ }^{16}$ which could also prove useful in generating FCA acceptance rate scenarios.

\section{Scheduling using a Probabilistic Capacity Forecast}

With the background given in Section II, the formal problem statement is given in Section III.A. The proposed approach to solving this problem is outlined in Section III.B, with details of this method following in Sections III.C through III.G. Concluding this section, advantages and disadvantages are summarized in Section III.H.

\section{III.A. Problem Statement}

Using the capacity forecast framework discussed in Section II, the problem can be stated as:

Given several possible capacity profiles for weather avoidance routes through an FCA with associated probabilities, find airport departure rates and airborne holding to satisfy capacity constraints at the FCA while minimizing the expected value of the delay cost. 


\section{III.B. Approach}

The schedule of departures and airborne holding for flights going to an FCA should be updated throughout the day to react to changing weather conditions. Most scheduling methods, e.g. Ref. 3, replan at a fixed frequency. A contribution of this paper is that a cost-benefit analysis determines when a departure replan should be implemented. In fact, AFPs are currently only updated if the update saves at least 10 minutes per flight, so a variation of this cost-benefit approach is already in use. Repeated changes to departure times cause aggravation for passengers and airlines and costly logistical changes. The value $\Gamma$ is the reduction of cost that is required for a departure replan to be implemented. It can be chosen to ensure that any departure rate update results in average delay savings greater than some specified minimum.

Replanning is done before the end of the accurate forecast horizon to ensure the satisfaction of constraints. At each time step, the weather forecast is updated and two separate linear programs (LPs), which will be formally defined later, are solved. Problem 1 generates departure rates and airborne holding. Problem 2 uses a fixed departure rate and generates airborne holding. As discussed in Section III.E, the cost that is to be minimized by both LPs is the expected value of the weighted sum of ground holding cost and airborne holding cost. The planning horizon ranges from time step $K_{I}$ to $K_{F}$. Both problems find solutions starting at the current time step $K$, where $K \geq K_{I}$, through $K_{F}$. They are solved at some frequency, $K_{R}$, where $K_{R}$ is less than or equal to the accurate forecast horizon, $\hat{K}$. Both $K_{R}$ and $\hat{K}$ have units of time steps. The algorithm proceeds as described here:

Input: FCA capacity profiles with associated probabilities, flight schedule, accurate forecast horizon $\hat{K}$, and replan frequency $K_{R}$ (where $\left.K_{R} \leq \hat{K}\right)$

Output: Departure rates and airborne holding

while $K \leq K_{F}$ do

Solve Problem 1 for departure rates and airborne holding for the time period $K$ to $K_{F}$;

Solve Problem 2 for airborne holding only for the time period $K$ to $K_{F}$, using departure rates

from previous loop ( $K_{R}$ time steps ago);

if $\operatorname{cost}$ (Problem 1$)+\Gamma<\operatorname{cost}($ Problem 2) then

| return departure rates and airborne holding from Problem 1;

end

else

| return departure rates from previous iteration and airborne holding from Problem 2;

end

$K=K+K_{R}$

end

The integration of the proposed departure rate and airborne holding scheduling method with weather prediction methods and the NAS is depicted in a flow chart in figure 2. The proposed scheduling method is represented by the red block titled "Generate TFM Control." Terms $c^{s}$ and $p^{s}$ represent the FCA capacity forecast and its associated probability of occurring, respectively, for each weather scenario, $s=1, \ldots, S$. The controlled departure rate and airborne holding control denoted by $d$ and $u$, respectively.

The departure rates are constrained to be the same for each weather scenario both during and beyond the accurate forecast horizon. This is done so that estimated departure times can be assigned for flights scheduled to depart after the accurate forecast horizon.

The airborne holding solution for each weather scenario is constrained to be identical for each weather scenario for the duration of the accurate forecast. Beyond the accurate forecast horizon, the airborne holding solutions for each weather scenario are allowed to differ. This results in the generation of departure rates and airborne holding that can actually be implemented for the accurate forecast horizon. Beyond this horizon the capacity of the FCA is uncertain, so the solution includes finding airborne holding for each weather scenario, which is then incorporated into the expected value of the cost function. In this way, the cost of future control is incorporated into the cost function and thus affects the departure rates and airborne holding calculated at the current time step.

\section{III.C. Airspace Model and Variable Definitions}

The model framework, associated variables and nomenclature are introduced in this section to provide context for the variables that will be used in the formal statements of Problems 1 and 2. This section gives a 


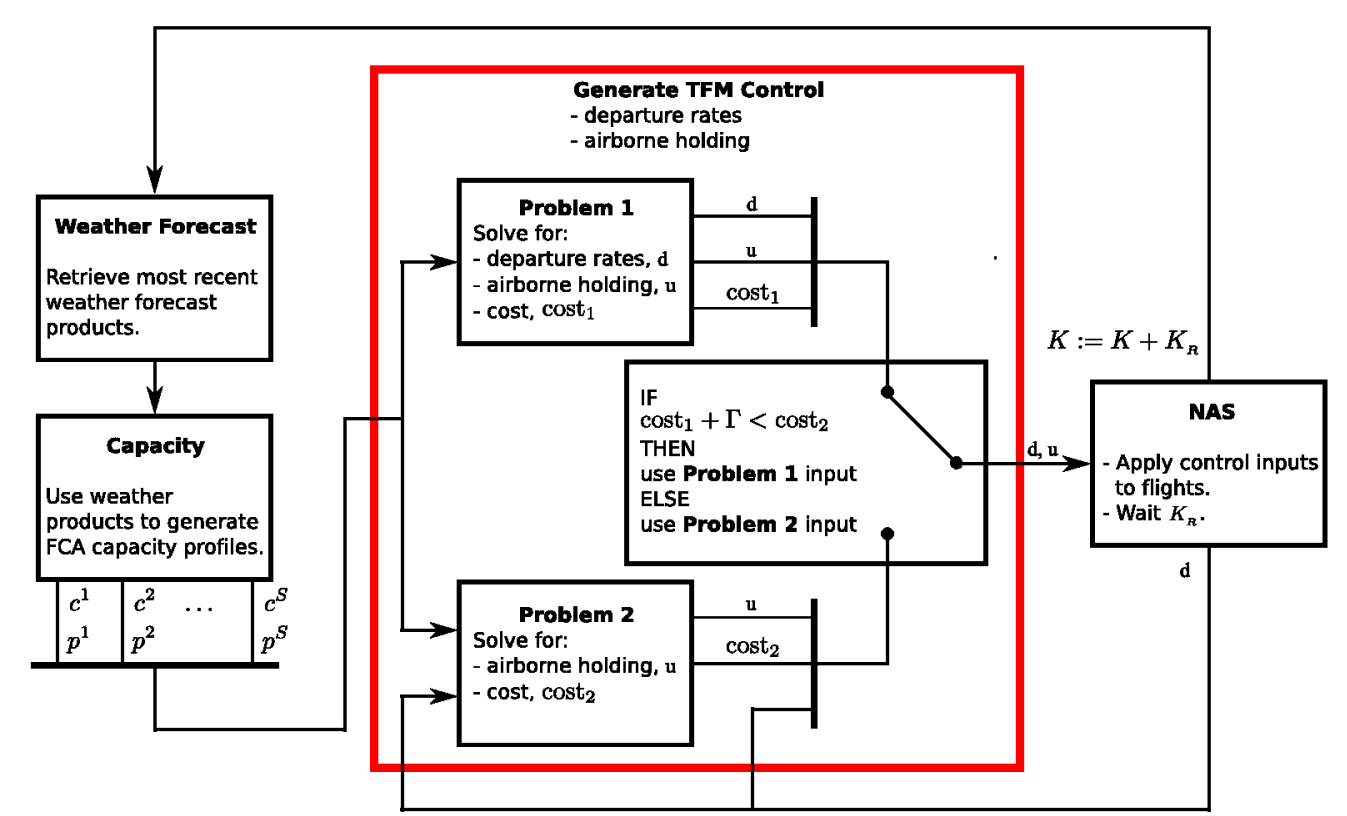

Figure 2. Chart showing the flow of information and TFM actions between weather forecast methods, capacity prediction methods, the NAS and the proposed method of scheduling departure rates and airborne holding control (represented by the block outlined in red).

high level overview of network dynamics and variable names. Equations relating these variables are stated as constraints in Problems 1 and 2 in Section III.D. These constraints ensure that the flow of aircraft through the network of sections leading to the FCA is physically achievable and feasible for the given set of FCA capacity constraints. Details of these constraints will be given in Sections III.F and III.G.

Airspace and flights are described mathematically by a discrete time, aggregate flow model. One of the advantages of using an aggregate model is that it can be described with linear equations. The equations can be used as constraints in a convex optimization problem, as is done in this work. Several aggregate flow models have been proposed with four such models compared in Ref. 17. The model used for this research is similar to the cell transmission model. ${ }^{5}$ In the model, routes between airports and the FCA are divided into sections. These sections could be existing sectors or other volumes of airspace. Figure 3 shows a simple example.

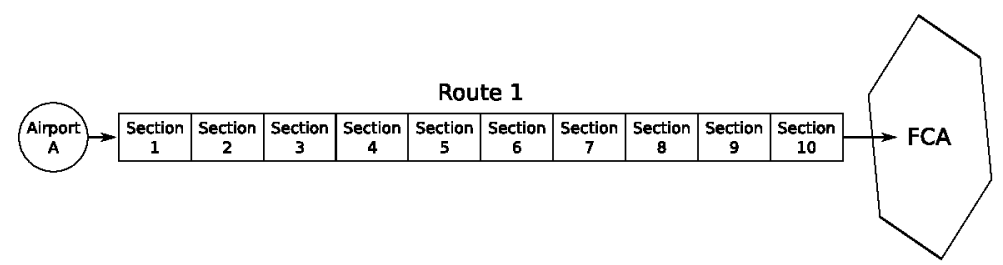

Figure 3. One airport problem: Aircraft depart from a single airport and fly to the FCA using a single route.

The length of a time step is represented by $\Delta t$. All aircraft are assumed to travel at the same average speed within each section, and thus the traversal times, $\tau_{i}, i=1, \ldots, N$, for each section specify how many time steps all flights require to traverse each section. Scheduled airborne holding can be thought of as requiring certain flights to remain in a given section longer than the average flight time. Airborne holding can be realized in the real system by decreasing flight speed, commanding a vector for spacing which increases the length of the flight path, or placing flights into holding patterns. Aircraft take off from the departure airport at the controlled rate $d$ and enter section 1. Aircraft flow from one section to the next sequentially within the route. Aircraft exiting the final section of the route, $N$, enter the weather avoidance route through the FCA. The term $c^{s}(k)$ represents the predicted flow rate capacity of the weather avoidance route during time step $k$ under weather scenario $s$. 


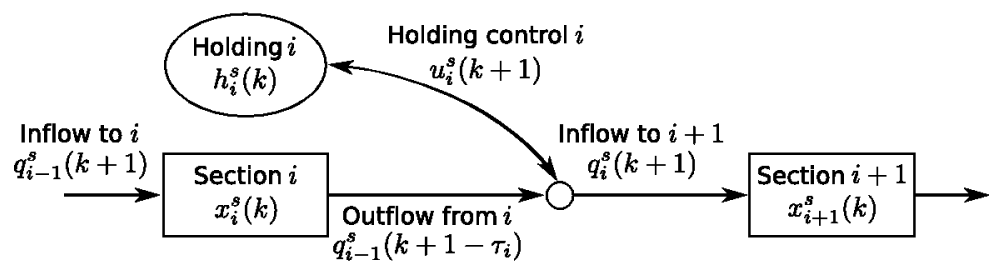

Figure 4. Variables and dynamics associated with the flow of aircraft at a typical section.

The flow of aircraft through a typical section of the route is presented graphically in figure 4. Subscripts of variables represent the index of a section. Superscripts represent the index of a capacity forecast. The time step is indicated within the parentheses. The quantities $x$ and $h$ represent the number of aircraft in a section and holding at a section, respectively. The quantities $u$ and $q$ represent the number of aircraft flowing from the given section into or out of holding $(u)$ and from one section to another $(q)$. For example, the term $x_{i}^{s}(k)$ represents the number of aircraft in section $i$ during time step $k$ under weather scenario $s$. It is assumed that both section and holding capacities are limited, with $\bar{x}_{i}$ the maximum allowable capacity for section $i$ and $\bar{h}_{i}$ the maximum allowable holding capacity at section $i$.

Equations which describe the flow of material between sections and holding and ensure the feasibility of solutions will be given as constraints of Problems 1 and 2 and will be explained in more detail in Sections III.F and III.G.

\section{III.D. Statement of LPs}

In this section, Problems 1 and 2 are formally defined. These problems are formulated as linear programs designed to minimize delay subject to uncertain airspace capacity constraints. Here, the uncertainty in airspace capacity constraints is explicitly incorporated by generating airborne holding beyond the accurate forecast horizon for each possible weather scenario. The airborne holding schedule determined for each scenario is included in the cost by taking the expected value of the airborne holding cost over all possible scenarios. More details of the formulation of the cost function are given in Section III.E.

This cost is minimized subject to dynamic and feasibility constraints. Dynamic constraints describe the physical flow of aircraft between sections and holding areas. The feasibility constraints ensure that control actions are achievable and that airspace capacity constraints are not violated. Detailed descriptions of dynamic and feasibility constraints are given in Sections III.F and III.G.

The control inputs available using this model framework are holding control at each section under each weather scenario, $u_{i}^{s}(\cdot)$ for all $i$ and $s$, and the departure rate at the airport, $d(\cdot)$. Problems 1 and 2 find $u_{i}^{s}(\cdot)$ and $d(\cdot)$ to minimize the expected value of delay costs while satisfying dynamic and feasibility constraints.

\section{Problem 1}

Given

$$
\begin{aligned}
& c^{s}(k), k=K, \ldots, K_{F}, \forall s \\
& \mathcal{D}(k), k=K_{I}, \ldots, K_{F} \\
& \bar{x}_{i}, \bar{h}_{i}, \forall i
\end{aligned}
$$

Find

$$
\begin{aligned}
d(k), k & =K, \ldots, K_{F} \\
u_{i}^{s}(k), k & =K, \ldots, K_{F}, \forall i, \forall s
\end{aligned}
$$

Minimize Cost (see Section III.E for details)

$$
w_{g}\left(\sum_{k=K_{I}}^{K_{F}} \sum_{l=K_{I}}^{k}[\mathcal{D}(l)-d(l)]\right) \Delta t+w_{a} \sum_{s=1}^{S} p^{s}\left(\sum_{k=K_{I}}^{K_{F}} \sum_{i=K_{I}}^{N} h_{i}^{s}(k)\right) \Delta t
$$


Subject To For each $s=1, \ldots, S$

Dynamic Constraints (see Section III.F for details)

$$
\begin{aligned}
h_{i}^{s}(k+1) & =h_{i}^{s}(k)+u_{i}^{s}(k+1), & i=1, \ldots, N, k & =K, \ldots, K_{F} \\
q_{1}^{s}(k+1) & =d\left(k+1-\tau_{1}\right)-u_{1}^{s}(k+1), & k & =K, \ldots, K_{F} \\
q_{i}^{s}(k+1) & =q_{i-1}^{s}\left(k+1-\tau_{i}\right)-u_{i}^{s}(k+1), & i=2, \ldots, N, k & =K, \ldots, K_{F} \\
x_{1}^{s}(k+1) & =x_{1}^{s}(k)+d(k+1)-d\left(k+1-\tau_{1}\right) & k & =K, \ldots, K_{F} \\
x_{i}^{s}(k+1) & =x_{i}^{s}(k)+q_{i-1}^{s}(k+1)-q_{i-1}^{s}\left(k+1-\tau_{i}\right), & i=2, \ldots, N k & =K, \ldots, K_{F}
\end{aligned}
$$

Feasibility Constraints (see Section III.G for details)

$$
\begin{aligned}
\sum_{l=K_{0}}^{k} \mathcal{D}(l)-\sum_{l=K_{0}}^{k} d(l) & \geq 0 \\
u_{i}^{s}(k) & =u_{i}^{1}(k) \\
-h_{i}^{s}(k) \leq u_{i}^{s}(k+1) & \leq q_{i-1}^{s}\left(k+1-\tau_{i}\right), \\
-h_{1}^{s}(k) \leq u_{1}^{s}(k+1) & \leq d\left(k+1-\tau_{1}\right), \\
0 \leq x_{i}^{s}(k) & \leq \bar{x}_{i} \\
0 \leq h_{i}^{s}(k) & \leq \bar{h}_{i} \\
q_{N}^{s}(k) & \leq c^{s}(k),
\end{aligned}
$$

$$
\begin{aligned}
k & =K, \ldots, K_{F} \\
k & =K, \ldots, K+\hat{K}, \forall i \\
k & =K, \ldots, K_{F}, i=2, \ldots, N \\
k & =K, \ldots, K_{F} \\
k & =K, \ldots, K_{F}, \forall i \\
k & =K, \ldots, K_{F}, \forall i \\
k & =K, \ldots, K_{F}
\end{aligned}
$$

\section{Problem 2}

Given

$$
\begin{aligned}
c^{s}(k), k & =K, \ldots, K_{F}, \forall s \\
\mathcal{D}(k), k & =K_{I}, \ldots, K_{F} \\
d(k), k & =K, \ldots, K_{F} \\
\bar{x}_{i}, \bar{h}_{i}, \forall i &
\end{aligned}
$$

\section{Find}

$$
u_{i}^{s}(k), k=K, \ldots, K_{F}, \forall i, \forall s
$$

\section{Minimize}

Equation (1).

Subject To For each $s=1, \ldots, S$

Dynamic Constraints

Equations (2) to (6).

Feasibility Constraints

Equations (8) to (13).

Problem 2 is similar to Problem 1 with only the parameters and variables differing between the two problems. Note that constraint (7) is not included in Problem 2 since this constraint is assumed to be satisfied by the given departure rate, $d(\cdot)$.

The number of variables in each of these problems depends on the number of sections used to model the airspace, the number of airports, and the number of weather scenarios considered. However, all equations are linear, which leads to less complex problems than those encountered when using Lagrangian methods. Also, dual decomposition methods can be used to solve these optimization problems relatively quickly. ${ }^{18}$ Finally, since an AFP is designed to control air traffic scheduled to fly through a specific region of airspace, only a subsection of the NAS needs to be modeled.

Actually, the solutions to these problems must be integers because only integer number of aircraft can depart or be in a region of airspace at a given time. This constraint is ignored in this research, but if this approach were implemented in a more realistic simulation of the NAS, such as the Future ATM Concepts Evaluation Tool (FACET), ${ }^{19}$ in which control inputs must be generated for individual flights, rounding heuristics could be applied or the problems could be posed as integer programming problems. 


\section{III.E. Cost}

Typically, the cost used for similar TFM problems is the weighted sum of ground and airborne delay. ${ }^{3,4}$ Weighted delay is used to account for the additional costs associated with airborne delay, such as the cost of additional fuel consumed. In Problems 1 and 2 a single departure rate profile is found for all capacity profile scenarios, resulting in a deterministic ground holding cost. Since airborne holding is generated for each scenario, a deterministic airborne holding cost does not exist. Therefore, the overall cost is an expected value that is expressed as

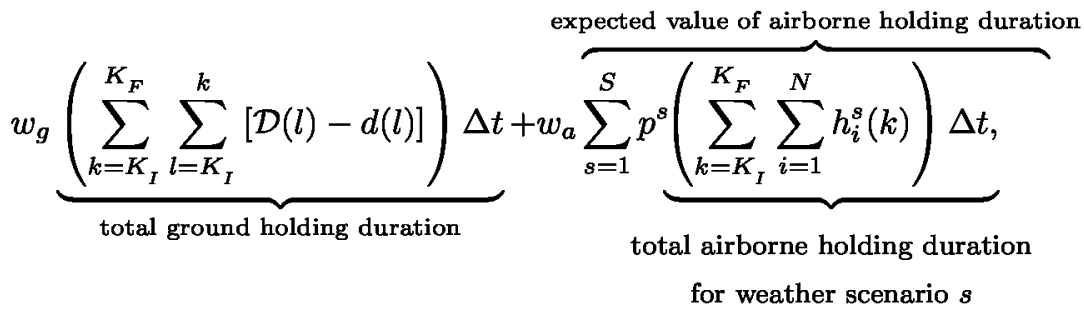

where $K_{I}$ and $K_{F}$ are the initial and final time steps of the full plan, respectively, $w_{g}$ and $w_{a}$ are the ground and airborne holding weights, respectively, $p^{s}$ is the probability that weather scenario $s$ will occur, and $S$ is the total number of weather scenarios given for the weather avoidance route. Note that it is required that $\sum_{s=1}^{S} p^{s}=1$ and $p^{s} \geq 0$ for all $s$.

As is commonly done in the literature, e.g. Ref. 3 , values of $w_{g}=1$ and $w_{a}=2$ roughly represent the fact that airborne holding is more expensive. Airborne holding costs airlines both time and money due to additional fuel consumption. Most TFM literature has used a $w_{a}$ value of 2 but without providing justification. Airline operating cost data from table 4-1 of Ref. 20 suggests that this ratio was 1.18 in 2002. More recent research ${ }^{21}$ suggests that, due to increases in fuel costs, the ratio was about 1.96 in 2006 . If fuel costs continue to increase or if airlines are charged for emissions, the ratio could become even higher.

\section{III.F. Dynamic Constraints}

In this section, the details of the dynamic constraints, equations (2) - (6), will be discussed. The dynamics of a typical section (index $i=2, \ldots, N$ ) under weather scenario $s$ are described first. The dynamics of section 1 are slightly different than a typical section since the inflow to this section depends on the departure rate. Thus, section 1 dynamics are described separately.

For a specific weather scenario, $s$, the number of aircraft in section $i$ during time step $k$ is $x_{i}^{s}(k)$. The number of aircraft flowing into section $i$ from an upstream section $i-1$ during time step $k+1$ is $q_{i-1}^{s}(k+1)$. The number of aircraft flowing out of section $i$ during time step $k+1$ is the number of aircraft that entered that section during time step $k+1-\tau_{i}$, i.e., $q_{i-1}^{s}\left(k+1-\tau_{i}\right)$.

Of the aircraft exiting section $i$, some number may enter holding at section $i$ while others will either enter section $i+1$ if $i=2, \ldots, N-1$ or the FCA if $i=N$. If $u_{i}^{s}(k+1)>0$, the control variable $u_{i}^{s}(k+1)$ represents the net number of aircraft exiting section $i$ that are sent into holding at section $i$. If $u_{i}^{s}(k+1)<0$, this value represents the net number of aircraft in holding at section $i$ that are removed from holding and sent on to section $i+1$ if $i=2, \ldots, N-1$ or to the FCA if $i=N$. The control input of aircraft sent into or removed from holding at a given section represents the net change in the number of aircraft holding at that section. For example, even if $u_{i}^{s}(k+1)=0$, some aircraft exiting section $i$ may be sent into holding while the same number of aircraft are removed from holding and sent on to section $i+1$, resulting in a net change in aircraft holding at section $i$ of 0 aircraft. The variable $h_{i}^{s}(k+1)$ represents the number of aircraft holding at section $i$ during time step $k+1$, the dynamics of which are described by Eq. (2).

The number of aircraft exiting section $i$ or leaving holding at section $i$ and entering either the subsequent section if $i=2, \ldots, N-1$ or the FCA if $i=N$ during time step $k+1$ is described by Eq. (4). Thus the number of aircraft in section $i$ at the end of time step $k+1$ is given by Eq. (6).

The flow of aircraft into and out of section 1 is defined by the departure rate at the airport. Therefore, Eq. (3) describes the number of aircraft exiting section 1 or leaving holding at section 1 and entering section 2 during time step $k+1$. Similarly, Eq. (5) gives the number of aircraft in section 1 at the end of time step $k+1$. 


\section{III.G. Feasibility Constraints}

The feasibility constraints, equations (7) - (13), of Problems 1 and 2 are discussed in more detail in this section. These constraints ensure that control actions are feasible, section and holding capacities are not exceeded, and the FCA flow rate capacity is not exceeded.

Equation (8) forces the airborne holding control to be identical for every weather scenario as long as the weather is assumed to be accurate $(\hat{K})$. These controls can then be implemented until the next replan occurs, which is required to happen before the end of the accurate weather prediction horizon.

The total number of controlled departures at the end of time step $k$ must not exceed the total number of aircraft originally scheduled to depart by the end of time step $k$. This avoids assigning a departure time that is earlier than the time at which this flight will be available for departure. This constraint can be represented mathematically by the inequality in Eq. (7), where $\mathcal{D}(l)$ represents the number of aircraft originally scheduled to depart from the airport during time step $l$.

The number of aircraft that are placed into holding at section $i$ during time step $k+1$ must not exceed the outflow of that section, $q_{i-1}^{s}\left(k+1-\tau_{i}\right)$, and the number of aircraft that are removed from holding during time step $k+1$ must not exceed the total number of aircraft holding in that section during time step $k$, $h_{i}^{s}(k)$. These constraints are captured by the inequalities in Eq. (9) and (10).

Since the state of each section represents the number of aircraft in that section, the value of $x_{i}^{s}(k)$ must be nonnegative for all $i, k$, and $s$. Additionally, due to limitations in the number of aircraft that can be safely routed through any particular section, a maximum capacity constraint can be enforced for each section. Both of these constraints are captured in the inequality in Eq. (11), where $\bar{x}_{i}$ is the maximum allowable capacity of section $i$. Note that $\bar{x}_{i}$ could be made dependent on the weather scenario but that in this work the weather scenarios are assumed to only impact the FCA, not the airspace around the FCA.

Similarly, the term $h_{i}^{s}(k)$ represents the number of aircraft in holding at section $i$ during weather scenario $s$ at the end of time step $k$ and must be a nonnegative value. Note that since

$$
u_{i}^{s}(k+1)=h_{i}^{s}(k+1)-h_{i}^{s}(k)
$$

by a rearrangement of Eq. (2), the constraints in Eq. (9) and (10) include the constraint that the number of aircraft holding at each section must be nonnegative, i.e. $h_{i}^{s}(k+1) \geq 0$. The maximum holding capacity at each section represents the number of aircraft that can safely be held at each section. This constraint is represented by the inequality in Eq. (12), where $\bar{h}_{i}$ is the maximum allowable holding capacity at section $i$. This capacity is set to zero at sections where holding is not possible. Also, it is possible to define an upper bound on the sum of $x_{i}^{s}(k)$ and $h_{i}^{s}(k)$ rather than bounding them individually, but this was not done in this research. The values of the maximum section and holding capacities can be chosen based on constraints specific to the region of airspace affected by the AFP and weather conditions of the AFP. Thus the selection of these capacities provides an additional level of control design.

For each weather scenario, the number of aircraft exiting section $N$ and entering the FCA under weather scenario $s$ during time step $k$ must not exceed the predicted flow rate capacity of the weather avoidance route through the FCA under weather scenario $s$. This flow rate constraint is captured by Eq. (13), where $c^{s}(k)$ represents the predicted capacity of the weather avoidance route during time step $k$ under weather scenario $s$.

\section{III.H. Advantages and Disadvantages}

Several properties of this proposed solution method are worth noting. As discussed previously, this approach requires that a change in departure rates induce cost savings of more than $\Gamma$. The value of $\Gamma$ may be chosen to ensure that the delay cost savings are not exceeded by implementation costs. This would require an estimate of airline specific costs, which may be difficult or impossible to calculate, depending on operation cost information that each airline is willing to share.

The strategy will not exceed the FCA capacity. The probabilistic cost of future control is considered. More likely weather scenarios will impact the control actions more than less likely scenarios. These features can be incorporated into solutions for many TFM problems, and they are considered contributions of this research.

A limitation of this approach is that intra-airline flight substitutions in the AFP are only guaranteed to be permissible if the two flights being substituted originate at the same airport and enter the FCA at the same weather avoidance route. These substitutions give airlines flexibility to use the arrivals to an 
FCA as they prefer. If flights originating at different airports or entering the FCA at different weather avoidance routes exchange their arrival times, the constraints on the capacity of the airspace sections and the weather avoidance routes may be violated. In order to accommodate flight substitutions of this type, each substitution would have to be evaluated and permitted if it does not lead to any constraint violations.

\section{Simulations and Results}

Results are presented comparing the proposed probabilistic approach to methods using different deterministic capacity profiles. Results for a one airport problem are presented in Section IV.B and results for a two airport problem are given in Section IV.C. Simulations were also performed to study the effects of varying the replanning frequency and replanning cost, $\Gamma$, with results presented in Section IV.D.

Simulations are performed for a number of weather events, where each event consists of a set of three possible capacity profiles and associated probability of occurring. In Section IV.A, the generation of these weather events and creation of deterministic capacity profiles for these events are described. Additionally, parameters common to each simulation are given at the end of the section.

\section{IV.A. Simulation Parameters and Setup}

The process of generating synthetic capacity predictions for the FCA proceeds as follows. Each synthetic capacity profile consists of three stages, a decreasing capacity stage, constant low capacity stage and increasing capacity stage. The low capacity value of each profile was randomly selected from a uniform distribution of the integers from 2 to 8 aircraft per time step. The low capacity values for each profile were required to be distinct. The duration of the decreasing and increasing stages were independently randomly chosen from a uniform distribution of the integers from 4 to 7 time steps. The duration of the minimum stage was randomly selected from a uniform distribution of the integers from 7 to 9 time steps. The start time of the decreasing stage was randomly selected from a uniform distribution of integer values while ensuring that the entire weather event occurred within a 24 time step block starting at time step 11. A typical set of three capacity profiles for the weather avoidance route through the FCA is given in figure 1. As labeled in figure 1 , the profiles will be referred to as the high, mid, and low capacity profiles.

In an effort to compare the proposed probabilistic approach, simulations were performed using the iterative approach discussed in Section III.B with approaches that use single profile FCA capacity forecasts for planning. When using a single capacity profile to represent the predicted capacity of the weather avoidance route, the expected value of the cost used in Problems 1 and 2 becomes a deterministic value. The results of these simulations are given in Sections IV.B and IV.C.

For each weather event used in these simulations, airport departure rates and airborne holding were planned using the method presented in Section III using one of the following capacity forecast methods: high, mid, low, expected value, probabilistic and constant. The high, mid, and low capacity forecast methods simply use the high, mid or low capacity profile as the predicted capacity. The expected value forecast uses the expected value of the capacity at each time step based on the three capacity profiles and their associated probabilities of occurring; an example is shown in figure 5. The method presented in Section III of using all three capacity profiles and minimizing the expected value of the cost is referred to as the probabilistic method. The constant capacity profile is constant throughout the duration of the weather event. This constant value is the average of all three capacity profiles for the average duration of the weather event; an example profile is shown in figure 5 . The use of a constant capacity is motivated by current AFP practices in which the default procedure is to set a fixed constant capacity. Changes to the FCA predicted capacity are made only if significant cost reduction can be achieved by adjusting the FCA capacity.

When solving the problem using one of these single profile methods, the set of possible FCA capacities, $c^{s}(\cdot)$ for $s=1, \ldots, S$ consisted of a single profile, thus $S=1$. When using the probabilistic method, all three profiles were used, each of which had an equal probability of occurring.

A set of 10 randomly generated weather events was used in these simulations in order to capture a small variety of weather conditions. When solving the problem using a specific weather event, one of three possible capacity profiles was chosen to be the "actual capacity profile." That is, one capacity profile was chosen to be the actual capacity profile of the FCA. Since all capacity profiles have equal probability, for each weather event, three simulations were performed. Each capacity profile, high, mid and low, was used as the actual weather profile in one of these three simulations. This was done to generate average results over all 

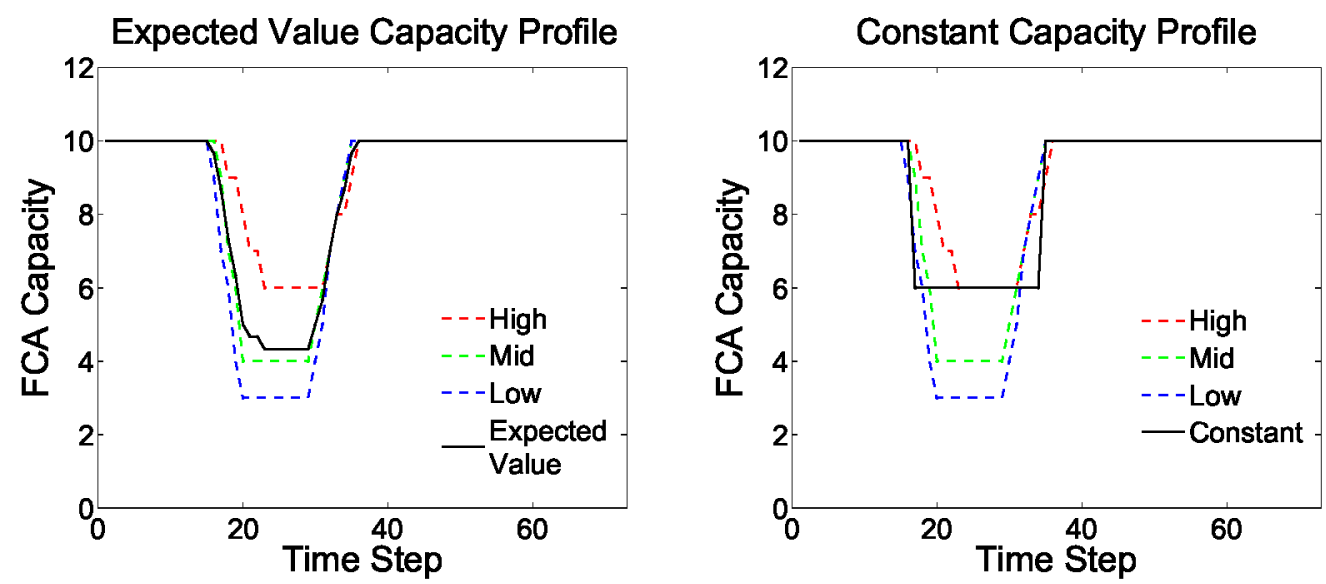

Figure 5. Predicted FCA capacity profle using the expected value approach (left) and the constant capacity approach (right).

capacity profiles that could occur. Future research should consider the more realistic scenario where the actual capacity of the FCA does not exactly match any forecast. For each solution, departure rates and airborne holding were scheduled.

For each actual weather scenario, there is an optimal solution within the modeling and control framework used. The optimal solutions are obtained when the predicted FCA capacity matches the actual FCA capacity exactly, i.e. the predicted FCA capacity is accurate for the entire planning horizon. The optimal solutions use ground holding only and the FCA capacity is matched precisely throughout the planning horizon. As a method of normalizing results, costs are reported in terms of a percent of this optimal cost.

The parameters common to each of the simulations are given in table 1. Parameters specific to each simulation will be given in the appropriate results sections.

Table 1. Parameter values used for simulations discussed in Sections IV.B, IV.C and IV.D.

\begin{tabular}{ll} 
Parameter & Value \\
\hline Time step: & $\Delta t=10$ minutes \\
Traversal time: & $\tau_{i}=1$ time step, $\forall i$ \\
Flight time from airport to FCA: & 10 time steps \\
Total number of departing flights: & 360 \\
Total simulation time: & 72 time steps \\
Section and holding capacity: & not enforced
\end{tabular}

\section{IV.B. One Airport Problem Results}

Using the problem setup consisting of a single airport and single weather avoidance route through the FCA, shown in figure 3, several simulations were performed to study the behavior of the proposed solution method. The problems use the parameters listed in table 1 . Additionally, the originally scheduled departure rate from the airport, $\mathcal{D}$, is 10 aircraft per time step for the first 36 time steps (or 1 aircraft per minute for 6 hours), dropping off to 0 aircraft per time step for the rest of the planning horizon. As shown in figure 3, the route leading from the airport to the FCA consists of 10 sections. The replanning cost is 0 , so new departure rates are implemented whenever calculated. Simulations were performed to compare the proposed probabilistic approach to several single capacity profile prediction methods, each of which is described in Section IV.A.

The results are presented in table 2. For a given capacity prediction method and actual capacity profile, the "Average Cost as Percent of Optimal" is the average cost incurred divided by the average optimal cost for the 10 events simulated. The "Overall Average Cost as Percent of Optimal" for each prediction method is the average cost, over all 10 events using each of the 3 capacity profiles as the actual capacity profile, divided by the average optimal cost for the same set of events. Note that using the high capacity prediction 
method with the actual capacity being the high profile, the resulting average cost as a percent of optimal is $100 \%$. This is because the capacity prediction matched the actual capacity for the entire planning horizon and the optimal solution was found.

Table 2. Average results from capacity model comparison simulations.

\begin{tabular}{|c|c|c|c|c|c|c|}
\hline $\begin{array}{c}\text { Capacity } \\
\text { Prediction } \\
\text { Method } \\
\end{array}$ & $\begin{array}{c}\text { Actual } \\
\text { Capacity } \\
\text { Profile } \\
\end{array}$ & $\begin{array}{l}\text { Average } \\
\text { Ground } \\
\text { Holding } \\
\end{array}$ & $\begin{array}{c}\text { Average } \\
\text { Airborne } \\
\text { Holding } \\
\end{array}$ & $\begin{array}{c}\text { Average } \\
\text { Cost }\end{array}$ & $\begin{array}{c}\text { Average Cost } \\
\text { as Percent } \\
\text { of Optimal }\end{array}$ & $\begin{array}{c}\text { Overall Average } \\
\text { Cost as Percent } \\
\text { of Optimal }\end{array}$ \\
\hline \multirow{3}{*}{ High } & High & 11155 & 0 & 11155 & 100.0 & \multirow{3}{*}{114.9} \\
\hline & Mid & 16074 & 2003 & 20080 & 116.5 & \\
\hline & Low & 20264 & 3596 & 27456 & 120.9 & \\
\hline \multirow{3}{*}{ Mid } & High & 17766 & 227 & 18220 & 163.3 & \multirow{3}{*}{122.5} \\
\hline & Mid & 17230 & 0 & 17230 & 100.0 & \\
\hline & Low & 22991 & 2078 & 27146 & 119.6 & \\
\hline \multirow{3}{*}{ Low } & High & 23497 & 218 & 23933 & 214.6 & \multirow{3}{*}{141.3} \\
\hline & Mid & 24366 & 586 & 25537 & 148.2 & \\
\hline & Low & 22701 & 0 & 22701 & 100.0 & \\
\hline Expected & High & 17078 & 68 & 17214 & 154.3 & \multirow{3}{*}{122.2} \\
\hline \multirow[t]{2}{*}{ Value } & Mid & 18496 & 593 & 19682 & 114.2 & \\
\hline & Low & 21950 & 1780 & 25510 & 112.4 & \\
\hline \multirow{3}{*}{ Probabilistic } & High & 16157 & 129 & 16415 & 147.2 & \multirow{3}{*}{116.2} \\
\hline & Mid & 17176 & 523 & 18222 & 105.8 & \\
\hline & Low & 20909 & 1900 & 24710 & 108.8 & \\
\hline \multirow{3}{*}{ Constant } & High & 17207 & 34 & 17276 & 154.9 & \multirow{3}{*}{129.3} \\
\hline & Mid & 19604 & 838 & 21280 & 123.5 & \\
\hline & Low & 23251 & 2124 & 27500 & 121.1 & \\
\hline
\end{tabular}

The "Average Cost as a Percent of Optimal" results are presented graphically in figure 6(a). From this chart, it can be seen that the probabilistic forecast method achieves a cost reduction of approximately $13 \%$ of the optimal cost over the constant capacity method, which corresponds to an average total delay reduction of 5.8 minutes per flight. It can also be seen that the high capacity profile prediction method outperforms the probabilistic method with a cost reduction of about $1.3 \%$ over the probabilistic method. It is interesting to note that when using the high prediction method, a larger portion of the cost is derived from airborne holding than any other method. This is because this prediction is optimistic and will allow more flights to depart which then must be held in the air when the actual FCA capacity is lower than originally predicted. All other capacity prediction methods incur the largest cost penalties when the high capacity profile is the actual capacity profile. However, comparing the high capacity prediction method to the probabilistic prediction method when the mid and low capacity profiles are the actual capacity profiles, there is a significant advantage, $11 \%$ and $12 \%$ of the optimal cost, respectively, to using the probabilistic prediction method. The corresponding average total delay reductions per flight are 1.0 and 2.9 minutes, respectively. While costs tend to be lower when more capacity is available, the cost penalty for not planning to fully take advantage of that capacity is a relatively large fraction of the cost that is achieved if all the capacity is used.

It can be concluded that, if probabilistic capacity forecasts are available, the use of the proposed scheduling scheme will lead to a cost reduction over the use of most of the methods studied here. The only single capacity profile method which out performs, on average, the proposed probabilistic method is the high capacity prediction method. However, in circumstances in which the actual capacity profile is the mid or low, the use of the probabilistic method can significantly reduce delay costs. Additionally, the high capacity prediction method assigns over twice the amount of airborne holding than the probabilistic method, on average.

Additional simulations were performed using randomly generated probabilities that each of the three scenarios would occur, as opposed to each scenario occurring with equal probability. For each weather event, the real weather scenario was selected using the associated probability distribution. The results were found to be similar to those presented in figure 6 . 


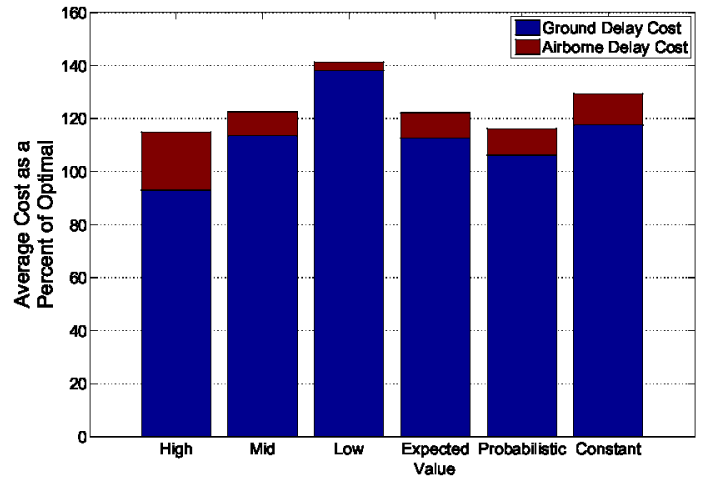

(a) Average results for all events and real weather scenarios

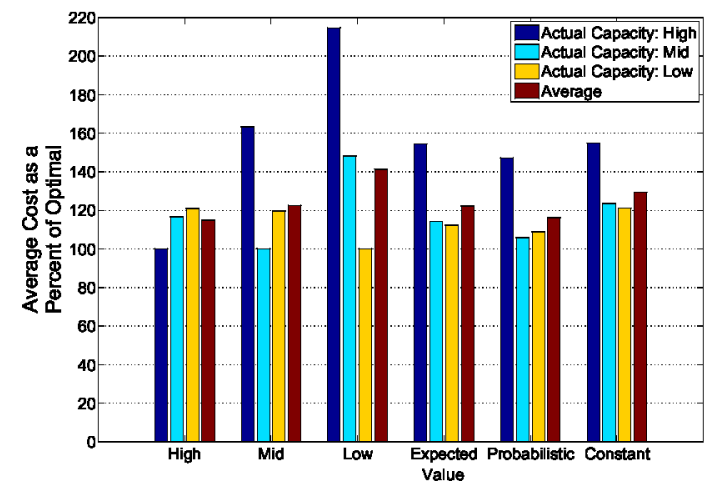

(b) Average results by real weather scenario type.

Figure 6. Cost comparison for several different capacity prediction methods for the one airport problem. In figure 6(a), average results over all events and all real weather scenarios are presented. In 6(b), average results over all events are broken down by the real weather profile type.

\section{IV.C. Two Airport Problem}

Simulations similar to those involving the one airport problem presented in Section IV.B were performed for a problem involving two airports and merging flow. A diagram of the specific network considered is shown in figure 7. The departure rate at airport $A$ is 7 aircraft every 10 minutes and 3 aircraft every 10 minutes at airport $B$. Departure rates at these airports were chosen so that the time history of the demand at the FCA is the same as that of the one airport problem. The same set of 10 events, with equal probability that each of the three capacity profiles will be the real capacity profile, as used in one airport problem were used for the two airport problem.

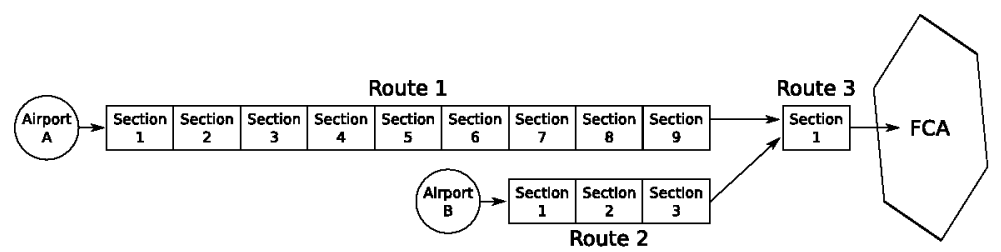

Figure 7. Two airport problem: Aircraft take off from airports $A$ and $B$ and fly towards the FCA using routes 1 and 2 respectively, and merge into route 3 before entering the FCA.

The average cost as a percent of optimal for each capacity prediction method is presented in figure 8(a). The data here exhibit the same trends as seen in the one airport problem, figure 6(a). However, for each capacity prediction method, costs are lower for the two airport problem than for the one airport problem. Solutions to the two airport problem use less airborne holding than solutions to the one airport problem. This is due to the fact that the departure rate at airport $B$ can be adjusted to react to changes in the FCA capacity prediction over a shorter time scale since flights departing from airport $B$ have a shorter nominal flight time to the FCA than those departing from airport $A$. In figure 8(b) average ground holding cost as a percent of optimal per flight is shown for each departure airport.

The issue of equitably distributing delay among flights arises in both AFPs and GDPs involving multiple departure airports. Several different scheduling paradigms for assigning delays to flights in an AFP and their effects on the solutions, including delay equity, are discussed in Ref. 2. At the aggregate level, equitable solutions will assign the same average delay per flight for all flights involved in the AFP, regardless of the respective distances from departure airports to the FCA. Notice that, although the high capacity prediction method results in an average cost which is lower than the average cost using the probabilistic method, the probabilistic method is more equitable, distributing ground holding more evenly to flights leaving from each of the two departure airports. Using the high capacity prediction method, average ground delay for flights departing from airport $B$ is $65 \%$ longer than flights departing from airport $A$, as opposed to only $20 \%$ when using the probabilistic capacity prediction method. 


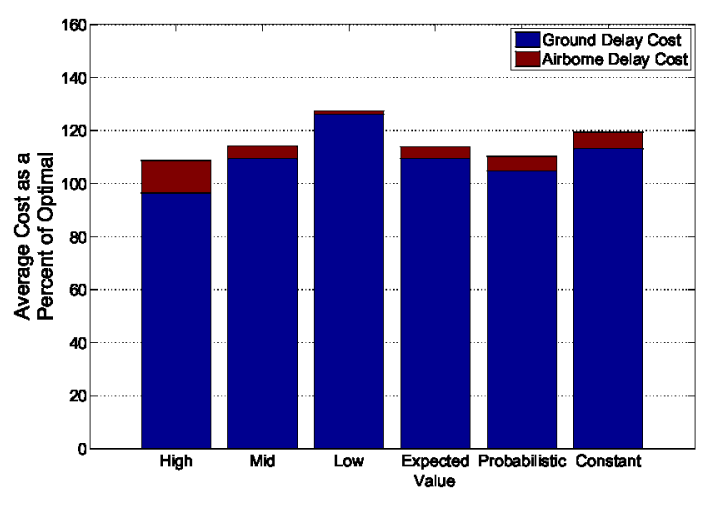

(a) Average results for all events.

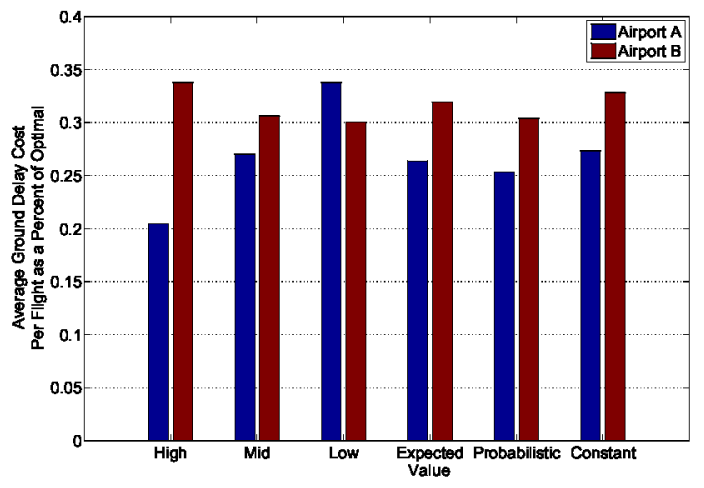

(b) Ground holding cost comparison.

Figure 8. Cost comparison for several different capacity prediction methods for the two airport problem. In figure 8 (a), average results over all events and all real weather scenarios are presented. In 8 (b), costs are shown per flight departing from airport $A$ and airport $B$.

\section{IV.D. Replanning Cost and Frequency Comparison}

In this section, replanning departure rates at a fixed frequency is compared to using a cost-benefit analysis to determine when departure rates should be updated. The one airport problem of figure 3 was used for this study. In addition to the parameters listed in table 1, the accurate forecast horizon is 120 minutes. Replanning frequencies of once every $30,60,90$, and 120 minutes were used for the fixed replanning frequency simulations. For these simulations, the replanning cost was set to 0 . Thus, new departure rates were implemented whenever calculated. Replanning costs, $\Gamma$, of $0,500,1000$, and 1500 were used with Problems 1 and 2 solved once every 30 minutes. In these simulations, airborne holding control could be updated every 30 minutes while departure rates were recalculated every 30 minutes, but actually implemented only if they resulted in a cost benefit greater than the replanning cost.

As described in Section IV.A, a set of three possible capacity profiles were randomly generated for each of ten weather events. Each capacity profile was given an equal probability of occurring. The probabilistic capacity prediction method was used for all simulations. As described in Section IV.A, three simulations were performed for each weather event in which each capacity profile, high, mid and low, was used as the actual capacity profile in one of these three simulations. The average cost as percent of optimal was generated for these simulations in the manner described in Section IV.B. Additionally, for each solution, the actual number of departure replans performed throughout the weather event were found. Note that even when replanning at a fixed frequency with no replanning cost, departure rates may not change from one replanning cycle to the next. Thus, when calculating the average number of departure replans, an actual departure replan is one in which the new departure rate plan differs from the previous departure rate plan.

In figure 9 , the average cost as a percent of the optimal is plotted versus the average number of departure replans for both the fixed replanning frequency method and the cost-benefit replanning method for cases in which the actual weather is the high, mid and low profile. There is a slight (1\% to $2 \%)$ improvement in cost when using the cost-benefit method to reschedule departure rates for the low and medium capacity actual weather profiles. For the high capacity profile, the cost savings as a percentage of the optimal cost are as high as $20 \%$ in some cases. This is partially because the optimal cost for the high capacity situation is lower to begin with, but also because replanning is not likely to be needed to avoid airborne holding as is the case when the lower capacity profiles occur. Also, note that if replan costs are added to the overall cost values, then the benefits of this cost-benefit replanning method would be more profound.

As mentioned earlier, it may be difficult to determine the appropriate value of $\Gamma$ to be used. However, the 10 minute per flight minimum delay reduction that is currently required for an AFP rate change could be used as a starting point. The operational use of this 10 minute threshold and the fact that an appropriate $\Gamma$ will reduce workload for all participants without sacrificing any cost suggest that it should be possible to arrive at a consensus for the $\Gamma$ value. 


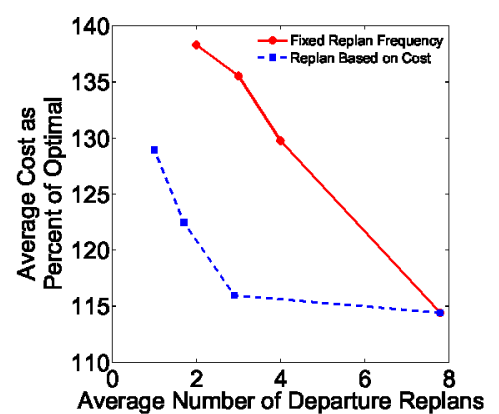

(a) Actual weather profile: High

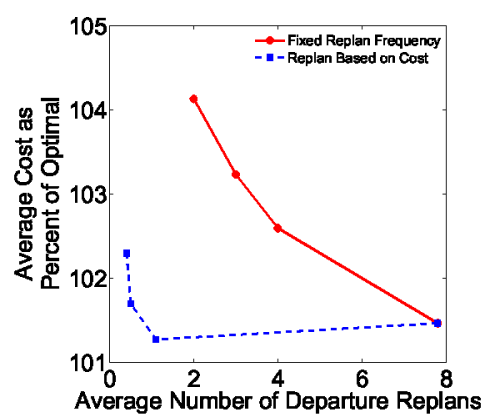

(b) Actual weather profile: Mid

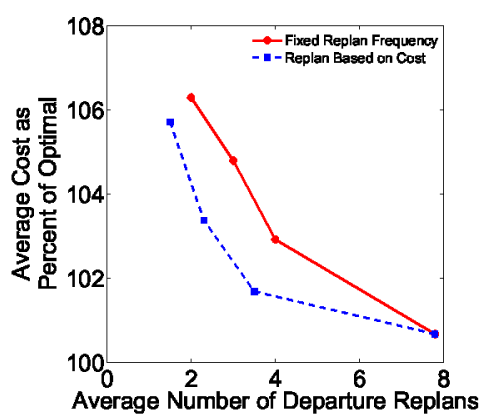

(c) Actual weather profile: Low

Figure 9. Cost of replanning departure rates at a fixed frequency (red circles) and based on a cost-benefit analysis (blue squares) as a function of the average number of departure replans. Note the variation in y-axis scales.

\section{Conclusions and Future Work}

A method for scheduling ground delay and airborne holding for flights scheduled to fly through an FCA with uncertain capacity has been proposed. The contributions of this approach are that it explicitly considers probabilistic constraint information, it only implements changes to the departure schedule when the resulting cost savings are sufficient to justify the change, and it takes advantage of new constraint information as it becomes available to ensure that capacity constraints are not violated. However, this approach does impose some restrictions on flight substitutions for flights involved in an AFP.

A variety of simulations have demonstrated the behavior of this approach. In these simulations, the constant capacity estimation method performed at $129 \%$ of the optimal cost level, while this approach was able to operate at $116 \%$ of the optimal cost. Similar performance is observed when this approach is applied to a two airport problem. Although the approach of assuming that the capacity will be high performs better than the probabilistic approach proposed here when considering cost only, there are some advantages to using the probabilistic approach. In the one airport problem, the high capacity prediction method assigns over twice the amount of airborne holding than the probabilistic method. The probabilistic method assigns delay more equitably in the two airport problem. Finally, a parametric study demonstrates that the cost-benefit feature of the proposed approach reduces the costs of the fixed planning frequency approach by between $1 \%$ and $20 \%$, depending on which capacity profile occurs.

Future work can be divided into theoretical, modeling and simulation work. More work must be done to understand why the approach of assuming that the capacity will be high performs better than the probabilistic approach in the simulations presented here. The primary future modeling work involves more complex networks of flows and multiple weather avoidance routes through FCAs. When considering these more complex problems, methods for disaggregating aggregate TFM controls into individual flight controls must be developed. When the flow network between airports and the FCA involves both merging and diverging, a mechanism for deciding which flights should take which network links must be developed. Similarly, when multiple weather avoidance routes through an FCA exist, a method for assigning flights to routes must be determined. Such methods should consider individual flight preferences.

The approach should be simulated with real traffic data from an actual AFP. Such a simulation would require implementation decisions such as the appropriate size for the airspace sections in the model and the disaggregation of control actions. Sun et al. present a method for disaggregation in Ref. 22 which addresses some of the issues that will be encountered when simulating the proposed control method with real traffic data.

\section{Acknowledgments}

The authors would like to thank Mark Evans (consultant), Al Mahilo (Supervisory Traffic Management Coordinator, Cleveland ARTCC), and David Foyle (Manager System Operations, Oakland ARTCC) for their insight into current AFP practices. Shon Grabbe and William Chan helped the authors understand current research related to weather-induced reductions in airspace capacity. The authors would also like to thank Cédric Langbort (Assistant Professor in the Department of Aerospace Engineering at the University 
of Illinois, Urbana-Champaign) for discussions regarding this research. This work was funded in part by a California Space Grant and NASA Aeronautics Scholarship Program Fellowship awarded to Heather Arneson.

\section{References}

${ }^{1}$ Bureau of Transportation Statistics, U.S. Department of Transportation, <http://www.bts.gov $>$.

${ }^{2}$ Hoffman, R., Burke, J., Lewis, T., Futer, A., and Ball, M., "Resource Allocation Principles for Airspace Flow Control," AIAA Guidance, Navigation, and Control Conference and Exhibit, 2005.

${ }^{3}$ Krozel, J., Jakobovits, R., and Penny, S., "An Algorithmic Approach for Airspace Flow Programs," Air Traffic Control Quarterly, Vol. 14(3), 2006, pp. 203-229.

${ }^{4}$ Bertsimas, D. and Patterson, S. S., "The Air Traffic Flow Management Problem with Enroute Capacities," Operations Research, Vol. 46, No. 3, May - June 1998, pp. 406 - 422.

${ }^{5}$ Sun, D. and Bayen, A. M., "Multicommodity Eulerian-Lagrangian Large-Capacity Cell Transmission Model for En Route Traffic," AIAA Journal of Guidance, Control, and Dynamics, Vol. 31, No. 3, May - June 2008, pp. 616 - 628 .

${ }^{6}$ Collaborative Decision Making, Federal Aviation Administration, "Collaborative Convective Forecast Product," <http://cdm.fly.faa.gov/products/ccfp1.html>.

${ }^{7}$ Avmet, "LAMP CCFP Hybrid" < http://www.lampccfp-hybrid.com>.

${ }^{8}$ DeLaura, R., Robinson, M., Pawlak, M., and Evans, J., "Modeling Convective Weather Avoidance in Enroute Airspace," Proc. of 13 th Conference on Aviation, Range, and Aerospace Meteorology, New Orleans, LA, 2008.

${ }^{9}$ Evans, J. E. and Ducot, E. R., "Corridor Integrated Weather System," MIT Lincoln Laboratory Journal, Vol. 16, No. 1, 2006, pp. 59-80

${ }^{10}$ Mitchell, J., Polishchuk, V., and Krozel, J., "Airspace Throughput Analysis Considering Stochastic Weather," $A I A A$ Guidance, Navigation, and Control Conference and Exhibit, Keystone, CO, August 2006.

${ }^{11}$ Krozel, J., Mitchell, J. S. B., Polishchuk, V., and Prete, J., "Maximum Flow Rates for Capacity Estimation in Level Flight with Convective Weather Constraints," Air Traffic Control Quarterly, Vol. 15, No. 3, 2007, pp. $209-238$.

${ }^{12}$ Prete, J. and Mitchell, J. S. B., "Safe Routing of Multiple Aircraft Flows in the Presence of Time-Varying Weather Data," AIAA Guidance, Navigation, and Control Conference and Exhibit, August 2004.

${ }^{13}$ Klein, A. and Cook, L., "Airspace Availability Estimation for Traffic Flow Management Using the Scanning Method," Proc. of AIAA/IEEE Digital Avionics Systems Conference, St. Paul, MN, October 2008.

${ }^{14}$ Ball, M. O., Hoffman, R., Odoni, A. R., and Rifkin, R., "A Stochastic Integer Program with Dual Network Structure and Its Application to the Ground-Holding Problem," Operations Research, Vol. 51, No. 1, January-February 2003, pp. $167-171$.

${ }^{15}$ Mukherjee, A. and Hansen, M., "A Dynamic Stochastic Model for the Single Airport Ground Holding Problem," Transportation Science, Vol. 41, No. 4, 2007.

${ }^{16}$ Liu, P., Hansen, M., and Mukherjee, A., "Scenario-based air traffic flow management: From theory to practice," Transportation Research Part B, Vol. 42, 2008, pp. 685-702.

${ }^{17}$ Sun, D., Strub, I. S., and Bayen, A. M., "Comparison of the Performance of Four Eulerian Network Flow Models for Strategic Air Traffic Management," Networks and Heterogeneous Media, Vol. 2, No. 4, December 2007, pp. 569 - 595.

${ }^{18}$ Sun, D., Large-scale modeling and optimization of en route air traffic flow, $\mathrm{PhD}$ thesis, University of California, Berkeley, August 2008 .

${ }^{19}$ Bilimoria, K., Sridhar, B., Chatterji, G., Sheth, K., and Grabbe, S., "FACET: Future ATM Concepts Evaluation Tool," 3rd USA/Europe Air Traffic Management R\&D Seminar, 2000.

${ }^{20}$ GRA, Incorporated, "Economic Values for FAA Investment and Regulatory Decisions, A Guide," Tech. Rep. Contract No. DTFA 01-02-C00200, FAA Office of Aviation Policy and Plans, Washington, DC, December 2004.

${ }^{21}$ DeArmon, J., Hoffman, J., Holden, T., Mayo, J., Solomos, G., Kuzminski, P., and Chambliss, A., "An Estimation of the Benefits of Air Traffic Flow Management," Proc. of International Council of the Aeronautical Sciences, Anchorage, AK, September 2008.

${ }^{22}$ Sun, D., Sridhar, B., and Grabbe, S., "Traffic Flow Management using Aggregate Flow Models and the Development of Disaggregation Methods," Accepted for presentation at AIAA Guidance, Navigation and Control Conference and Exhibit, Chicago, IL, August 2009 